\title{
Educação continuada em serviços de medicina nuclear: Capacitação da equipe multidisciplinar em tempos de COVID-19
}

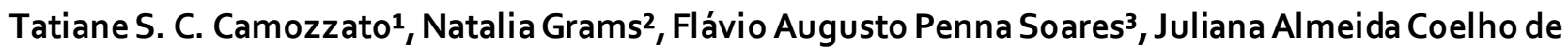 \\ Melo4, Itamara Almeida Cardoso5, Maria Júlia da Silva ${ }^{6}$
}

\begin{abstract}
Resumo: A modalidade de Ensino a Distância (EaD) busca facilitar o acesso e a democratização a todos que procuram por qualificação ou continuidade dos estudos. Aliada à extensão universitária, pode se tornar uma excelente ferramenta para promover a educação continuada nos ambientes de trabalho. O objetivo deste estudo foi relatar a experiência da equipe de docentes e discentes do Curso Superior de Tecnologia em Radiologia e do Mestrado Profissional em Proteção Radiológica do Instituto Federal de Santa Catarina em parceria com o Instituto de Cardiologia do Hospital Homero de Miranda Gomes, de São José/SC, durante a primeira oferta de um curso de capacitação via EaD para equipe multidisciplinar do serviço de Medicina Nuclear. Trata-se de um relato de experiência sobre o desenvolvimento e a operacionalização de um curso de extensão universitária. Dos 307 indivíduos inscritos no curso, 113 não chegaram a iniciá-lo, 77 participantes acessaram o cronograma de aula, assistiram ao vídeo de boas-vindas ao curso, mas não assistiram às videoaulas e tampouco realizaram alguma avaliação, e 116 alunos realizaram todas as avaliações propostas e concluíram o curso. Apenas 1 aluno realizou as avaliações e não atingiu a média 6,0. A ação de extensão promoveu a interação entre discentes da educação superior, da pós-graduação, docentes e comunidade externa em nível nacional e internacional. Dessa maneira, ocorreu a consolidação da cultura extensionista na educação superior e o despertar da pós-graduação para a necessidade de uma integração com a sociedade por meio da extensão.
\end{abstract}

Palavras-chave: Medicina Nuclear; Proteção Radiológica; Educação à Distância; Cursos de Capacitação

\section{Continuing education in nuclear medicine services: Training the multidisciplinary team in times of COVID-19}

Abstract: The Distance Learning modality (E-learning) seeks to facilitate access and democratization for all who seek qualification or continuity of studies. Combined with university extension, it can become an excellent tool to promote continuing education in the workplace. This study aimed to report the experience of the team of professors and students of the Higher Course in Technology in Radiology and the Professional Master's Degree in Radiological Protection at the Federal Institute of Santa Catarina (IFSC) in partnership with the Cardiology Institute of Hospital Homero de Miranda Gomes from São José, Santa Catarina, Brazil, during the first edition of a training course via distance learning for the multidisciplinary team of the Nuclear Medicine service. It is an experience report on the development and operation of a university extension course. Of the 307 individuals enrolled in the course, 113 did not start, 77 participants accessed the class schedule, watched the welcome video to the course, but did not watch the video classes or perform any assessment, 116 students performed all the proposed assessments completing the course. Only 1 participant performed the assessments and did not reach an average of 6.0. The outreach action promoted interaction between higher education students, graduate students, teachers, and the external community at national and international levels. Thus, the extension culture in higher education was consolidated, and the awakening of postgraduate courses to integrate with society through extension.

Keywords: Nuclear Medicine; Radiological Protection; E-learning; Training Courses
Originais recebidos em

10 dejunho de 2021

Aceito para publicação em

14 de setembro de 2021

1

Tecnóloga em Radiologia. Mestre em Ciências Biológicas: Fisiologia (UFRGS). Doutora em Ciências da Saúde (PUC-RS). Docente do Instituto Federal de Educação, Ciência e Tecnologia de Santa Catarina (IFSC). http://orcid.org/0000-0002-3530-718X (autora para correspondência) tatiane@ifsc.edu.br

2

Tecnóloga em Radiologia (IPUC)

Especialista em Imaginologia pela AVM-Faculdade Integrada; Especialista em Gestão e Auditoria em Sistemas da Saúde.

natalia.grams@ifsc.edu.br

3

Engenheiro Eletricista e Mestre em Processamento de Imagens, Doutor em Física Médica, Docente do IFSC.

flavio@ifsc.edu.br

4

Tecnóloga em Radiologia, Doutora em Enfermagem (UFSC), Docente do IFSC.

julianac@ifsc.edu.br

5

Técnica em Radiologia, Graduanda e bolsista de pesquisa e extensão do Curso Superior de Tecnologia em Radiologia (IFSC).

itamaraacardoso@gmail.com

6

Graduanda e bolsista de pesquisa e extensão do Curso Superior de Tecnologia em Radiologia (IFSC). mariajuliaasilva27@gmail.com 


\section{Introdução}

A modalidade de Ensino a Distância ( $\mathrm{EaD}$ ) busca facilitar o acesso e a democratização a todos que buscam uma qualificação ou continuidade dos estudos. Portanto, o acesso à Internet e à inserção das Tecnologias de Informação e Comunicação (TC) no cotidiano da sociedade proporcionaram a utilização de ambientes virtuais de aprendizagem (AVA) como ferramenta de ensino que pode conectar um maior número de pessoas, levando o conhecimento a lugares remotos e conectando discentes a docentes. Assim, possibilita-se o ensino, contribuindo para a educação continuada (Lisboa \& Rendeiro, 2019).

A pandemia, consequência da COVID-19, estabeleceu uma nova ordem para a humanidade, um isolamento necessário que impôs exercícios de cidadania. Consequentemente, as instituições de ensino sofreramimpactos e, assim, fez-se necessário o uso ampliado das tecnologias digitais, como o ensino remoto e a educação à distância, por exemplo. Logo, viu-se o potencial dessas tecnologias para a educação continuada, conectando profissionais de diferentes partes do país interessados em potencializar os saberes profissionais (Souza, 2020).

Dentre as diferentesáreas da saúde, destacamos neste estudo a Medicina Nuclear (MN). Trata -se de um campo da medicina cuja particularidade básica é a aplicação de emissores de radiação ionizante (radionuclídeo) em sua forma não selada, com a finalidade de tratamentos ou formação de imagem para diagnóstico. Os emissores de radiação formam os radiofármacos quando se vinculam a moléculas de interesse biológico, os fármacos (Camozzato et al., 2019).

Melo (2018) destaca que no campo de MN todos os profissionais de uma equipe multi e interdisciplinar são necessários e que esta equipe deve ser treinada para o trabalho com fontes de radiação não seladas. Os profissionais que atuam em um Serviço de Medicina Nuclear (SMN) são tecnólogos em radiologia, enfermeiros e técnicos de enfermagem, físicos, médicos e todos os colaboradores que não estão diretamente envolvidos, mas que são essenciais, como limpeza e recepção. Viana (2018) traz em seu estudo que os indivíduos ocupacionalmente expostos (IOE) na medicina nuclear trabalham diariamente no manuseio dos radionuclídeos e, portanto, recebem uma quantidade significativa de dose de radiação.

A prática dos SMN sofreu drásticas modificações nos últimos 10 anos devido ao surgimento de novas tecnologias. Essas mudanças trouxeram preocupação para a Agência Internacional de Energia Atômica (IAEA), que publicou um guia sistematizado das necessidades práticas da $\mathrm{MN}$ no que diz respeito à avaliação, às premissas, aos recursos humanos, a equipamentos e à garantia de qualidade e controle de qualidade, à física médica e ao suporte radiofarmacêutico, além de proteção radiológica e segurança e aplicações clínicas (International Atomic Energy Agency [IAEA], 2020).

A Portaria 3.05 CNEN NN (Comissão Nacional de Energia Nuclear [CNEN], 2013) traz como responsabilidade do titular do SMN a obrigatoriedade de treinamento de IOE, com periodicidade máxima anual, tanto para atuação em situações normais de trabalho quanto em situações de incidente ou acidente. Além disso, no anexo I, apresenta os tópicos mínimos para o treinamento de IOE em proteção radiológica, divididos em 3 tópicos: gerais, teóricos e práticos.

A Resolução CNE/CES nº 7 do Ministério da Educação (2018), que estabelece as Diretrizes para a Extensão na Educação Superior Brasileira, a destaca como interdisciplinar, incorporada à matriz curricular e à organização da pesquisa. A indissociabilidade do tripé ensino, pesquisa e extensão destaca o papel das instituições de ensino superior em promover ações que integrem a comunidade acadêmica e a externa (Cardenuto et al., 2016). Dessa maneira, a extensão universitária torna-se uma oportunidade única para modificar a sociedade na qual está inserida. 
Os projetos pedagógicos de curso devem alcançar, no mínimo, 10\% de atividades de extensão na matriz curricular dos cursos (Resolução CNE/CES n ${ }^{\circ}$ 7, de 18 de dezembro de 2018. Estabelece as Diretrizes para a Extensão na Educação Superior Brasileira e regimenta o disposto na Meta 12.7 da Lei $n^{\circ}$ 13.005/2014, 2018). Projetos extensionistas ampliam os conhecimentos, permitindo a aprendizagem teórico-prática, maximizando o ensino obtido em sala de aula (Musse et al., 2021). Além disso, os alunos envolvidos enriquecem o próprio conhecimento, tornando-se um ser mais crítico, com capacidade de organizar as ideias e torná-las concretas, a partir de novas concepções, novos costumes ou novos padrões comportamentais (Cardenuto et al., 2016).

A partir da curricularização da extensão no Instituto Federal de Santa Catarina (IFSC), surgiu a necessidade da implementação de ações de extensão, no dia a dia dos envolvidos com o ensino. Diante desse contexto, o objetivo deste estudo foi relatar as experiências da equipe de profes sores e discentes do Curso Superior de Tecnologia em Radiologia (CSTR) e Mestrado Profissional em Proteção Radiológica (MPPR) do Instituto Federal de Santa Catarina (IFSC), em parceria com o Instituto de Cardiologia do Hospital Homero de Miranda Gomes, de São José/SC, durante a primeira oferta de um curso de capacitação via EaD para equipe multidisciplinar em Medicina Nuclear.

\section{Metodologia}

Trata-se de um relato de experiência sobre o desenvolvimento e a operacionalização de um curso de extensão universitária. A equipe foi formada com a participação de 24 discentes do Curso Superior de Tecnologia em Radiologia (CSTR), 6 discentes do Mestrado Profissional em Proteção Radiológica (MPPR), 2 alunas bolsistas, além de 4 docentes do CSTR e MPPR do IFSC. A equipe teve um período de 6 meses para organizar o curso, redigir um livro digital, gravar as videoaulas, fazer divulgação, formulário de inscrição, além de tantas outras tarefas e, ainda, a o final do curso, fazer a análise dos dados obtidos por questio nários e formulários. Para tanto, foi necessária a divisão do projeto por fases.

O curso de Capacitação: Segurança e Proteção Radiológica para equipe multidisciplinar em SMN, foi realizado pelo IFSC, em Florianópolis, em parceria como Instituto de Cardiologia do Hospital Homero de Miranda Gomes em São José/SC. O projeto foi aprovado na modalidade Projetos de Integração da Pesquisa e Extensão ao Ensino do Câmpus Florianópolis, em conjunto com a Pró-Reitoria de Extensão e Relações Externas (PROEX), que é responsável por planejar, desenvolver e controlar os projetos de pesquisa e extensão no IFSC.

O curso foi disponibilizado integralmente de forma EaD, por meio da utilização do AVA, tendo seu início em 1 de março de 2021. As inscrições, que iriam do dia 1 a 28 de fevereiro de 2021, acabaram sendo antecipadas por conta da grande demanda que o curso teve, sendo encerradas no dia 15 de fevereiro. No total alcançamos 470 interessados. Destas 470 pré-inscrições, aceitamos apenas os alunos que se encaixavam no perfil do Público-Alvo: Profissionais da MN, estagiários em MN e profissionais da saúde, totalizando 307 inscritos.

\section{Fases do Projeto}

A primeira fase do projeto iniciou no mês de novembro de 2020 e foi necessário dividir os temas com a equipe colaboradora. Os grupos foram coordenados pelos professores orientadores do projeto, os quais foram responsáveis por realizar encontros com a equipe de forma não presencial, utilizando a plataforma Google

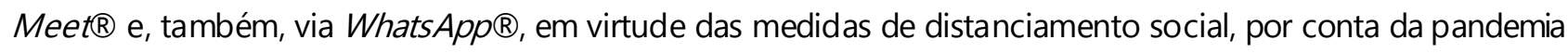
da COVID-19. Os encontros foram utilizados para planejamento das ações, elaboração da revisão de literatura dos assuntos abordados no curso, apresentação dos processos da MN, identificação das normas legais e dos riscos associados ao trabalho nos SMN. 
A segunda fase ocorreu entre os meses de novembro de 2020 a janeiro de 2021. Nessa fase foi realizada a elaboração e a correção, bem como a gravação de 22 videoaulas com duração de 3 a 10 minutos. As gravações ocorreram de forma não presencial e foram utilizados recursos como: câmera de celular e teleprompter, a

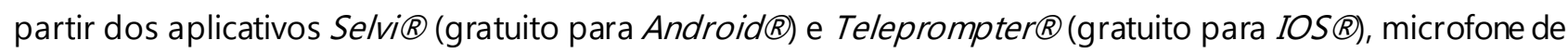
lapela ou fone de ouvido do celular e iluminadores (Ring light, luzes ou luminárias). A divisão dos módulos e videoaulas está apresentada na Figura 1.

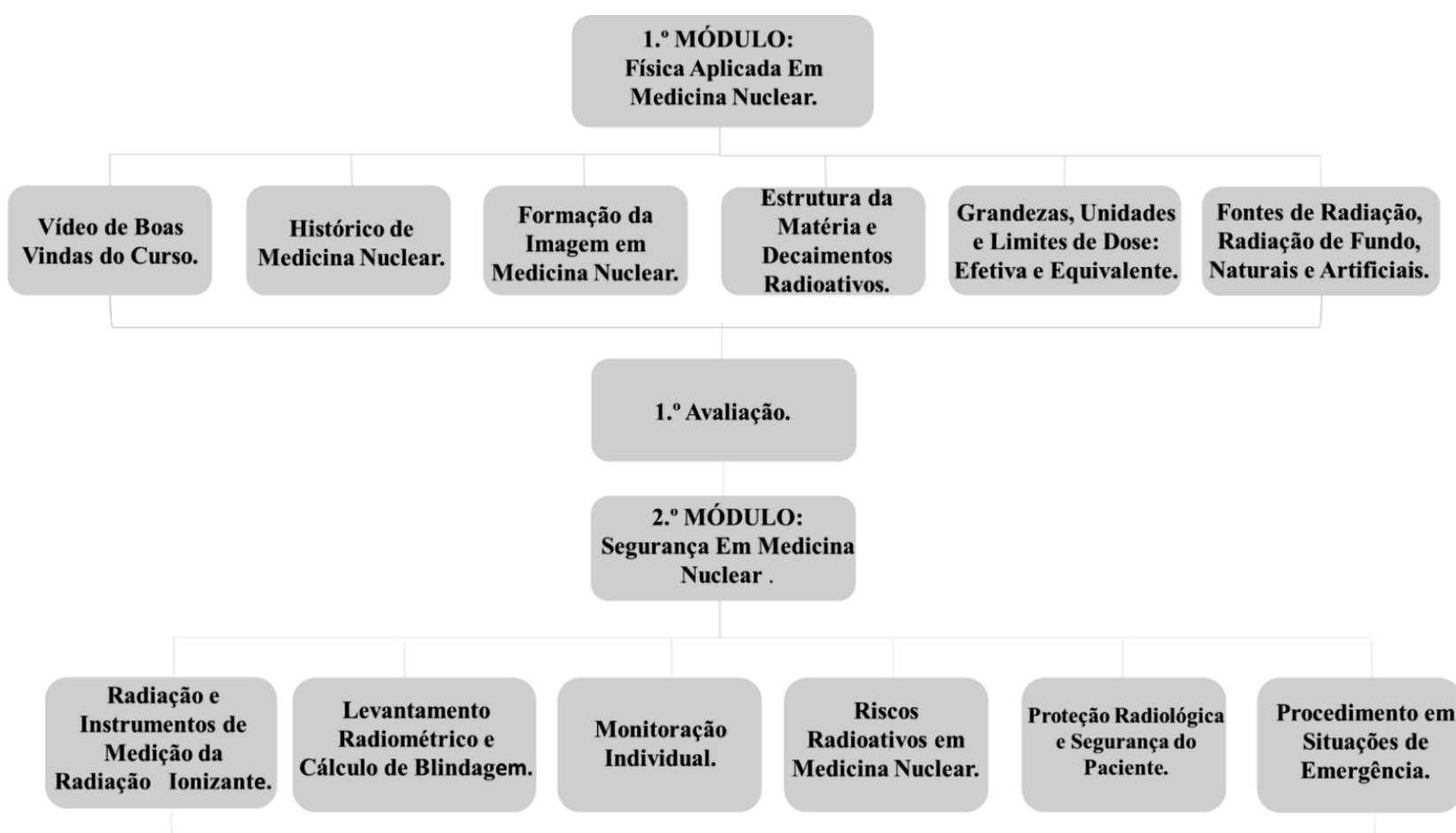

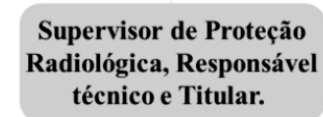

Supervisor de Proteção técnico e Titular.

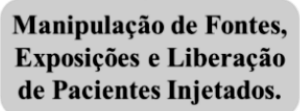

Manipulação de Fontes, de Pacientes Injetados.
Cuidados com Pacientes Injetados em Medicina Nuclear.

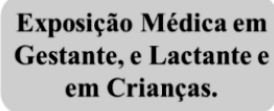

Exposição Médica em em Crianças.
Manuseio de Fontes Não Seladas e Gerenciamento de Rejeitos Radioativos.
Métodos de Descontaminação.

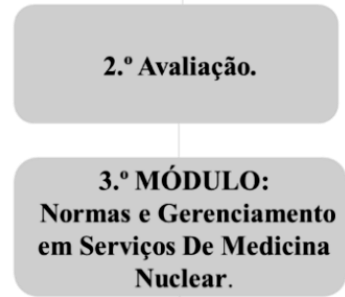

Nuclear. 
Nessa fase, também foi elaborado o cartaz de divulgação do curso, o formulário de inscrição pelo Google Forms ${ }^{\circledR}$ com questões para verificar o perfil dos alunos, além da criação de um e-mail para sanar quaisquer dúvidas em relação ao curso de capacitação. Foi realizada uma pesquisa no site da CNEN, no qual verificou-se que existem 466 instalações de medicina nuclear no Brasil. Ao fazer contato com todas as instalações autorizadas pela CNEN, 41 instalações responderam ter interesse no curso e passaram o endereço eletrônico dos seus supervisores de proteção radiológica.

O curso foi divulgado também em redes sociais com uso do cartaz produzido e da comunicação entre os inscritos. Dessa forma, houve divulgação nacional, a qual resultou em 470 inscrições. Para isso, foi necessário alterar a data do cartaz de divulgação e anunciá-lo novamente (Figura 2).

A terceira fase ocorreu nos meses de novembro de 2020 a fevereiro de 2021. Nessa fase foi realizada a elaboração de material pedagógico para compor um livro digital, com o intuito de servir como material de apoio aos estudantes de uma forma clara e objetiva. Foi necessário dividir os colaboradores em pequenos grupos de autores para dar início à elaboração do livro - assim, cada grupo de autores foi responsável, de acordo com a expertise dos orientadores, pelo desenvolvimento de um tema. Nesta etapa de correção houve a participação dos professores deste projeto e de revisores externos, de forma que a figura 3 apresenta a capa do livro digital.

Na quarta fase, as alunas bolsistas foram responsáveis pela criação do curso de capacitação no AVA institucional, que é a plataforma Moodle®. Nessa plataforma de ensino, realizaram a formatação dos módulos e aspectos visuais, o upload das videoaulas, cronograma de aula e materiais complementares, assim como a elaboração de questionários de avaliação na plataforma de ensino, sempre com auxílio dos docentes. O curso teve a duração de 46 dias, composto por 03 módulos, cujos conteúdos foram dispostos conforme demonstrado no fluxograma, e os quais foram liberados semanalmente, em consonância com o cronograma estabelecido, o que totalizou 7 semanas consecutivas.

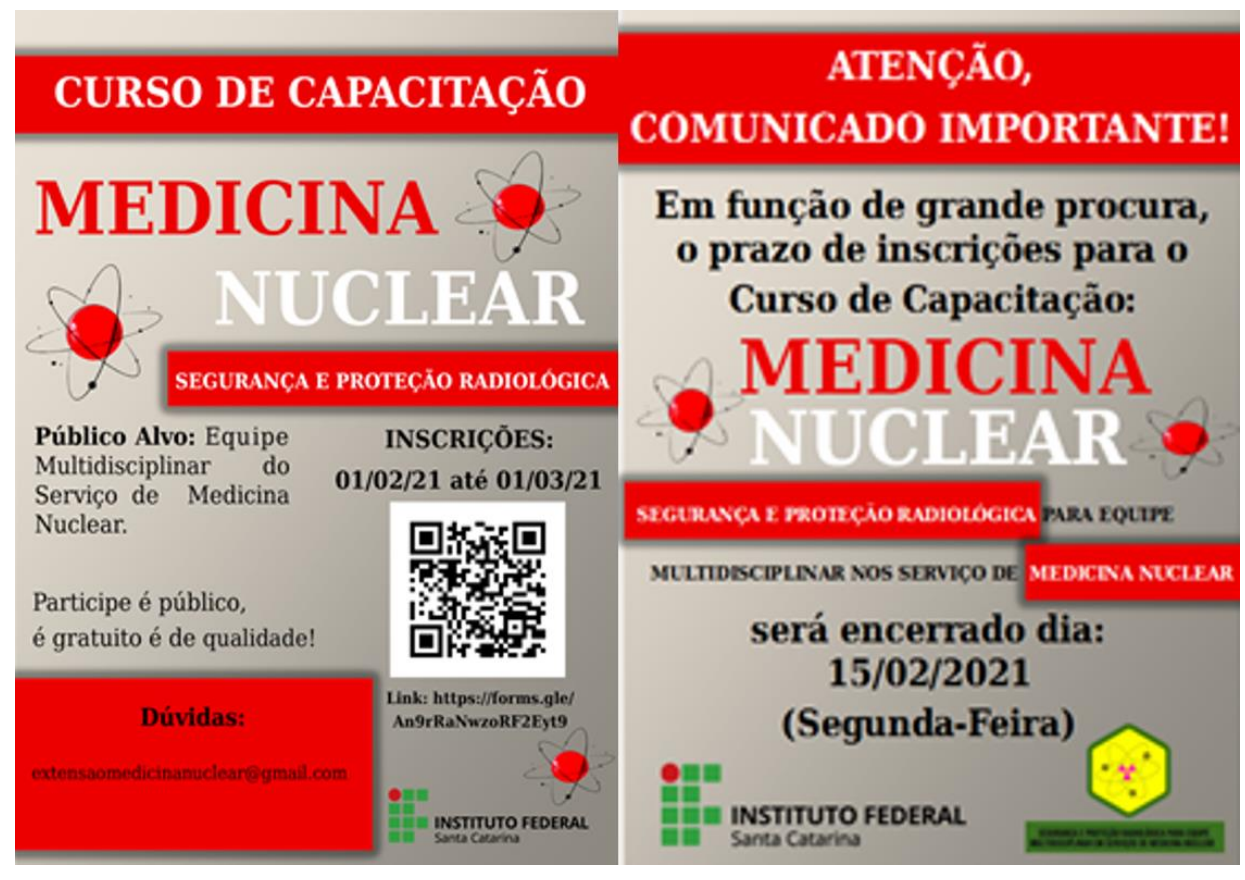

Figura 2. Cartaz de divulgação do Curso (à esquerda) e cartaz de divulgação do Curso alterado (à direita). 
Figura 3. Capa do livro digital. Fonte: Autores (2021).

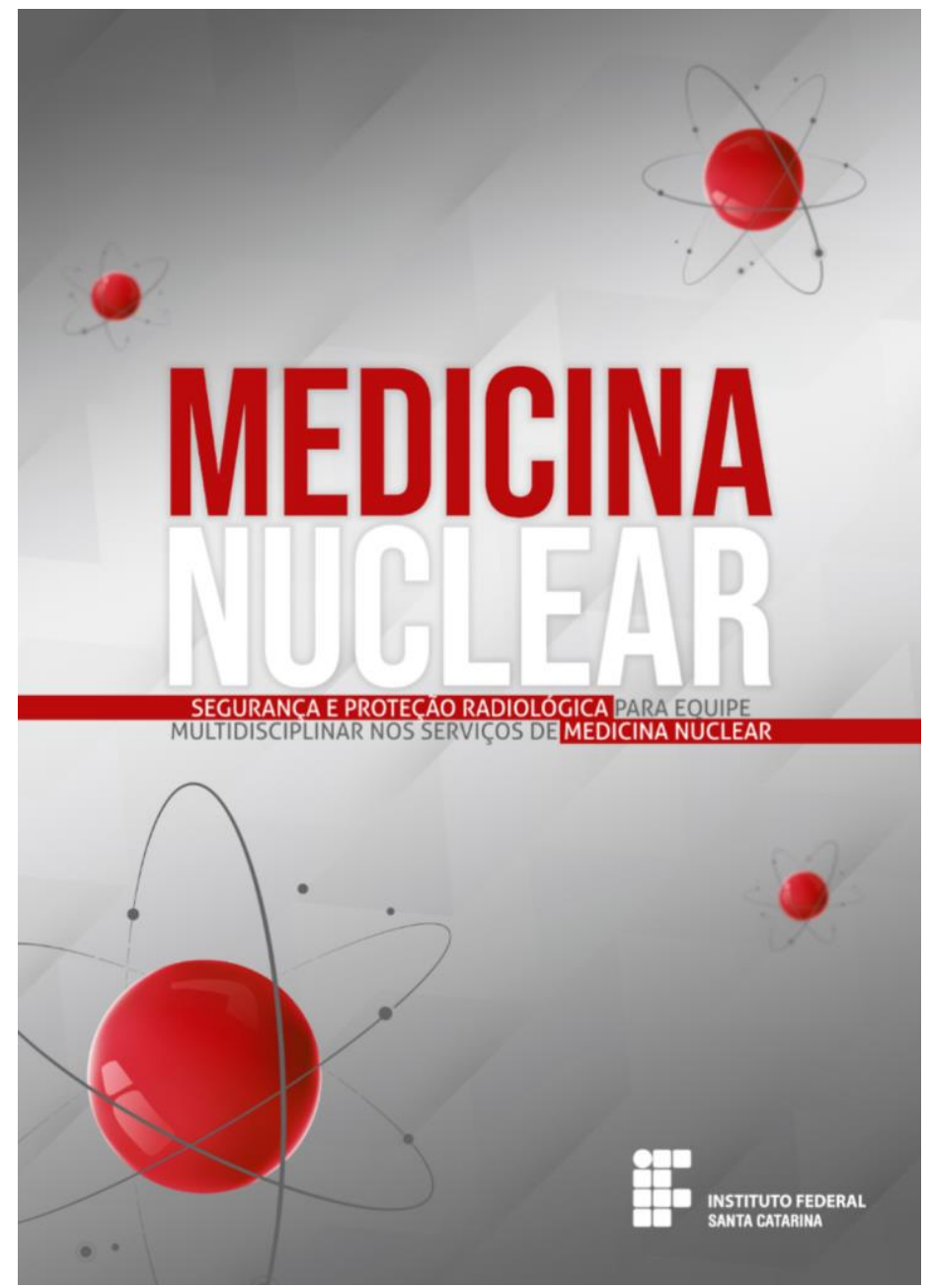

A quinta fase deste projeto refere-se à análise de dados em relação às perguntas respondidas no formulário de inscrição, ao aprendizado dos alunos durante o curso, no qual responderam a três questionários avaliativos e a uma pesquisa de satisfação. Os alunos que atingiram nota igual ou maior que seis nas três avaliações receberam o certificado de conclusão de curso. O processo de certificação ocorreu por meio da plataforma de Sistema Integrado de Gestão de Atividades Acadêmicas (SIGAA) do IFSC, onde foram cadastrados os alunos concluintes. Após esse cadastro, foi realizada a emissão dos certificados pelos docentes envolvidos no projeto, que os enviaram aos participantes por meio do endereço eletrônico. Após a análise dos dados, a equipe colaboradora do projeto analisou as respostas aos questionários avaliativos.

\section{Resultados}

\section{Análise do Perfil dos Alunos}

O perfil dos alunos do curso de capacitação foi obtido após análise do formulário de inscrição no curso, disponibilizado de forma online. Os inscritos responderam a perguntas relacionadas a sua formação, área de atuação, raça, gênero, escolaridade, entre outras perguntas.

Em relação ao perfil dos 307 alunos que efetivamente acessaram os conteúdos, verificou-se que os inscritos têm idade entre 19 e 69 anos, dos quais: $29 \%$ têm entre 19 e 29 anos; $41 \%$, entre 30 e 39 anos; 24\%, entre 40 
e 49 anos; e 6\%, entre 49 e 69 anos. A Figura 4 apresenta a distribuição dos participantes por regiões do país e nacionalidade.

Em relação à escolaridade, 65 dos inscritos (21\%) têm pós-graduação, 18 (6\%) concluíram o mestrado e 11 (4\%) possuem doutorado. Desses participantes com diversos níveis de escolaridade, as profissões que tiveram maior número de inscritos foram: técnicos e tecnólogos em radiologia, com 223 inscritos (73\%), seguido de 25 físicos (8\%), 17 técnicos em enfermagem e 9 enfermeiros (5\% e 3\%), 18 biomédicos (6\%), e o restante dos participantes eram de outras áreas da saúde.

Quanto ao perfil dos participantes, quando perguntados se já participaram de algum curso de capacitação em medicina nuclear, 191 participantes (62\%) responderam que não realizaram nenhum curso, 84 (27\%) responderam que sim e realizaram o curso na modalidade presencial, enquanto apenas $32(10 \%)$ relataram que sim e realizaram a capacitação na forma EaD.

Em relação à experiência em cursos de capacitação online, 232 (76\%) dos estudantes relataram possuir experiência e $24 \%$ não. Na ocasião também foi abordado sobre a facilidade que os alunos têm para estudar em um computador ou celular, a partir de que se constatou que 298 (97\%) possuem facilidade e apenas 9 (3\%) não possuemagilidade comessa modalidade de ensino; esses estudantes foram, então, monitorados de forma individualizada, no intuito de dirimir possíveis dúvidas que pudessem ter relação com a modalidade de ensino ou o uso do AVA.

Quando abordado se as videoaulas são eficazes para capacitar os profissionais do serviço de medicina nuclear, 292 (95\%) responderam que são eficazes e que as videoaulas contribuem para sua capacitação, enquanto 15 (5\%) responderam que não acham eficazes as videoaulas como meio para capacitar profissionais. Na ocasião, também foi questionado sobre a eficácia dos questionários avaliativos para capacitar os profissionais: cerca de 288 (94\%) responderam que sim - os questionários avaliativos são eficazes para a capacitação, enquanto 19 (6\%) responderam que não.

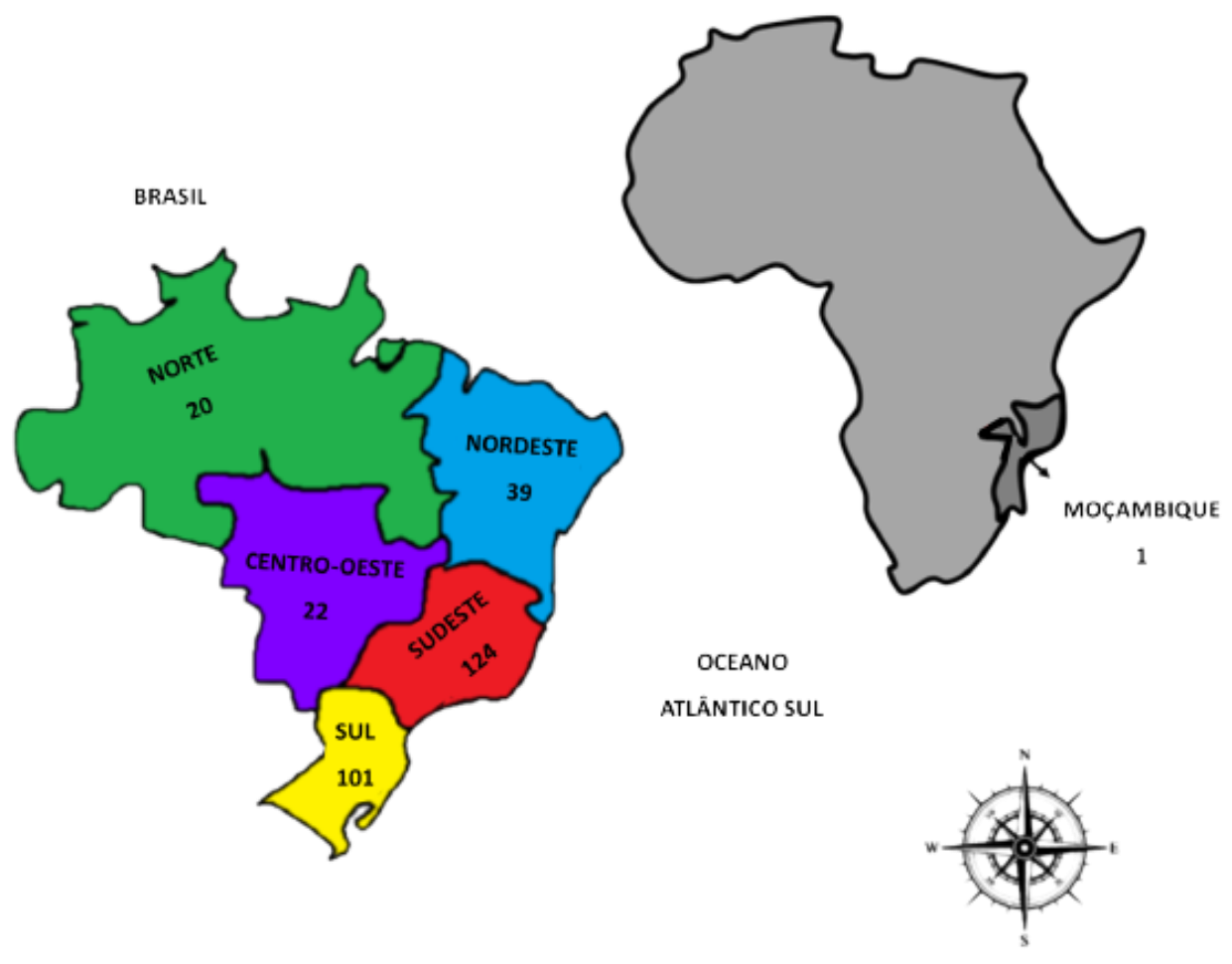

Figura 4. Mapa de Distribuição dos participantes por país e região. Fonte: Autores (2021). 
Em relação à Capacitação Profissional em MN, 285 inscritos (93\%) relataram que necessitam de capacitação nesta área, 23 (7\%) se sentem confiantes em relação ao seu conhecimento e responderam que não necessitam de capacitação.

Perguntamos aos inscritos como haviam tomado conhecimento da divulgação do curso resultando que 163 (53\%) responderam que foi devido ao compartilhamento em grupos de Whats $A p p \otimes, 87$ (28\%) souberam por

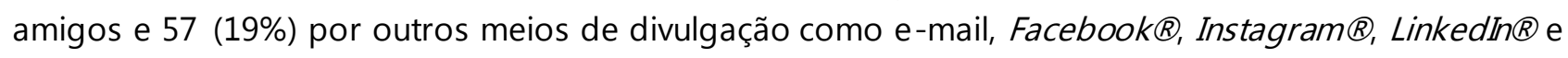
divulgação do cartaz no local de trabalho.

\section{Análise de dados}

A análise de dados demonstrou que, dos 307 inscritos, cerca de 194 (63\%) alunos acessaramo ambientevirtual de aprendizagem Moodle $\bigotimes_{\text {, enquanto }} 113$ (37\%) não o acessaram. Destes alunos que acessaram o ambiente Moodle $\AA$, 77 (25\%) alunos leram o cronograma de aula, assistiram ao vídeo de boas-vindas ao curso, mas não assistiram às aulas online, e também não realizaram as avaliações. Apenas 1 aluno reprovou e 116 concluíram o curso. Após uma semana do início, identificamos os participantes que ainda não haviam acessado a

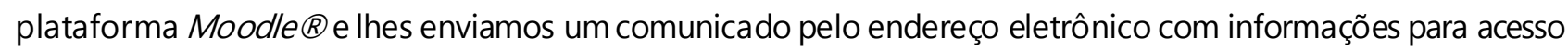
à plataforma de ensino.

A avaliação de desempenho dos alunos ocorreu em cada módulo do curso, após disponibilizadas aulas assíncronas, ou seja, aulas gravadas, que são a base de cursos EaD. O aluno foi obrigado a acessar todas as aulas e o material disponibilizado no curso para confirmar a sua participação e frequência. Foi disponibilizada, ao final do módulo, uma avaliação (AV), na qual o aluno só seria aprovado se atingisse nota superior a 6,0 nas avaliações. A Tabela 1 representa as notas separadas por país e região. A Figura 5 apresenta a pesquisa de satisfação em relação ao curso.

\section{Discussão}

O projeto Curso de Capacitação: Segurança e Proteção Radiológica para Equipe Multidisciplinar em Serviços de MN foi idealizado por alunos do MPPR do IFSC, durante a disciplina de Emergências Radiológicas, sugerido como uma estratégia para reduzir os acidentes radiológicos (Nascimento et al., 2018), por meio da capacitação da equipe multidisciplinar, que é responsável por manipular, administrar e adquirir as imagens dos exames da especialidade de MN (Portela et al., 2021).

A instituição de ensino, IFSC, que viabilizou o projeto, tem a extensão regulamentada pela Resolução $n^{\circ}$ 61/2016 do seu Conselho Superior (CONSUP). Apresenta essa atividade como "uma ação que promove a transformação social no entorno do campus, envolvendo servidores e discentes por meio de programas, projetos, cursos, eventos ou produtos" (IFSC, 2016). Esse projeto teve o envolvimento de discentes de mestrado, apesar de não estar previsto dentro do PPC desse curso (IFSC, 2014). Cabe destacar que as Diretrizes para a Extensão na Educação Superior Brasileira sugerem que as atividades a cadêmicas de extensão também podem ser direcionadas aos cursos superiores de pós-graduação (Resolução CNE/CES nº 7 do MEC, 2018).

Desde sua concepção, o objetivo do projeto foi proporcionar uma interação dialógica da comunidade acadêmica com setores sociais, especialmente com os profissionais da saúde, abordando temas relacionados à proteção radiológica e física aplicada em $M N$, segurança em $M N$ e normas e gerenciamento em SMN. A atividade segue as diretrizes da extensão para educação superior, entre as quais se destaca a troca de conhecimentos entre ambos, por meio da participação e contato com as questões complexas contemporâneas presentes no contexto social, bem como a possibilidade de proporcionar a mudança de hábitos e comportamentos por intermédio da disseminação do conhecimento e a articulação entre ensino/extensão/ pesquisa (Resolução CNE/CES nº 7 do MEC, 2018). 
Tabela 1. Informações dos Inscritos por País e Região. Fonte: Autores (2021).

\begin{tabular}{|c|c|c|c|c|c|c|c|c|}
\hline País e Região & Ins critos & Avaliação & $\begin{array}{c}\text { Nota } \\
\text { Mínima }\end{array}$ & $\begin{array}{l}\text { Nota } \\
\text { Máxima }\end{array}$ & Média & $\begin{array}{c}\text { Alunos } \\
\text { Aprovados }\end{array}$ & $\begin{array}{c}\text { Alunos } \\
\text { Reprovados }\end{array}$ & $\begin{array}{c}\text { Alunos } \\
\text { Desistentes }\end{array}$ \\
\hline Brasil & & AV 1 & 6,0 & 10,0 & 8,7 & & & \\
\hline \multirow{2}{*}{ Norte } & 20 & AV 2 & 10,0 & 10,0 & 10,0 & 7 (35\%) & 0 & $13(65 \%)$ \\
\hline & & AV 3 & 8,0 & 10,0 & 9,4 & & & \\
\hline Brasil & & AV 1 & 8,0 & 10,0 & 9,3 & & & \\
\hline \multirow{2}{*}{ Nordeste } & 39 & AV 2 & 8,0 & 10,0 & 9,8 & $12(31 \%)$ & 0 & 27 (69\%) \\
\hline & & AV 3 & 8,0 & 10,0 & 9,8 & & & \\
\hline \multirow{3}{*}{ Centro-Oeste } & & AV 1 & 7,0 & 10,0 & 9,2 & \multirow{3}{*}{13 (59\%) } & \multirow{3}{*}{0} & \multirow{3}{*}{9 (41\%) } \\
\hline & 22 & AV 2 & 8,0 & 10,0 & 9,5 & & & \\
\hline & & AV 3 & 10,0 & 10,0 & 9,7 & & & \\
\hline \multirow{3}{*}{ Sudeste } & & AV 1 & 6,0 & 10,0 & 9,2 & \multirow{3}{*}{48 (39\%) } & \multirow{3}{*}{0} & \multirow{3}{*}{76 (61\%) } \\
\hline & 124 & AV 2 & 6,0 & 10,0 & 9,3 & & & \\
\hline & & AV 3 & 6,0 & 10,0 & 9,6 & & & \\
\hline \multirow[t]{3}{*}{ Brasil } & & AV 1 & 4,0 & 10,0 & 9,1 & \multirow{3}{*}{36 (36\%) } & \multirow{3}{*}{$1(1 \%)$} & \multirow{3}{*}{64 (63\%) } \\
\hline & 101 & AV 2 & 4,0 & 10,0 & 9,4 & & & \\
\hline & & AV 3 & 6,0 & 10,0 & 9,6 & & & \\
\hline \multirow{3}{*}{ Moçambique } & & AV 1 & - & - & - & \multirow{3}{*}{$0(0 \%)$} & \multirow{3}{*}{-} & \multirow{3}{*}{1 (100\%) } \\
\hline & 1 & AV 2 & - & - & - & & & \\
\hline & & AV 3 & - & - & - & & & \\
\hline \multirow{4}{*}{$\begin{array}{c}\text { Brasile } \\
\text { Moçambique }\end{array}$} & & AV 1 & 4,0 & 10,0 & 9,1 & \multirow{4}{*}{$116(37,8 \%)$} & \multirow{4}{*}{$1(0,3 \%)$} & \multirow{4}{*}{$190(61,9 \%)$} \\
\hline & 307 & & & & & & & \\
\hline & (100\%) & AV 2 & 4,0 & 10,0 & 9,4 & & & \\
\hline & & AV 3 & 6,0 & 10,0 & 9,6 & & & \\
\hline
\end{tabular}




\section{Pescpuisa Satisfação}

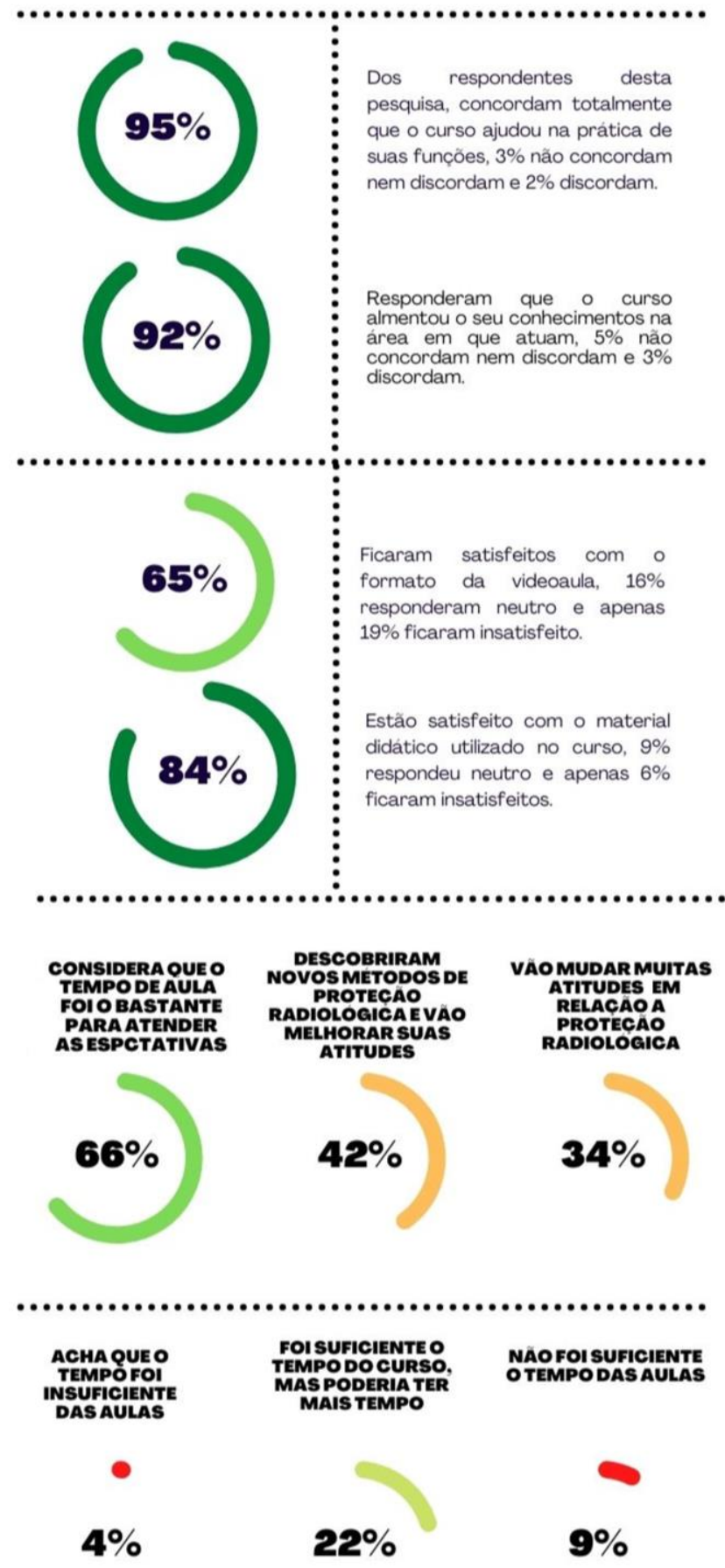

Figura 5. Pesquisa de Satisfação. Fonte: Autores (2021). 
Os cursos de educação superior e mestrado profissional envolvidos nessa ação de extensão são presenciais (IFSC, 2014, 2018); porém, durante a pandemia, tiveram todas as suas atividades desenvolvidas no formato "Atividade Não Presencial" (ANP). A legislação atual prevê que mesmo os cursos superiores na modalidade a distância realizem as atividades de extensão presencialmente, em região compatível com o polo de apoio presencial (Resolução CNE/CES nº 7 do MEC, 2018). Diante da realidade da pandemia, e sendo ne cessária a utilização da educação a distância, foi possível atingir um público maior do que no formato presencial de educação continuada, até mesmo com um aluno estrangeiro.

Dos 307 alunos inscritos no curso, cerca de 51\% têm preferência por cursos na modalidade online e 49\% preferem cursos na modalidade presencial. Segundo Santos (2020), as Instituições de Ensino Superior (IES) e a comunidade acadêmica estão se adaptando a essa nova realidade trazida pelas ANPs, construindo uma maior compreensão sobre as potencialidades sobre $\mathrm{a} \mathrm{EaD}$, que apres enta elementos como a utilização de plataformas digitais, videoconferências, ambientes virtuais de aprendizagem, por exemplo.

Conforme demonstraram Granjeiro et al. (2020), as ferramentas educacionais virtuais permitiram a realização de um trabalho inovador na formação inicial e continuada de alunos, professores e profissionais de saúde, proporcionando um ganho de conhecimento individual e coletivo. Assim sendo, é possível a ampliação do público assistido, já que existe uma flexibilidade nas atividades, pois o curso poderá ser ofertado em diversos momentos, haja vista que o conteúdo programático se encontra finalizado e o cursista não possuirá horários e dias fixos para acessar o material (Melo et al., 2020).

Dificuldades com o uso das tecnologias digitais e a falta de habilidades para as exigências da EaD podem ser demonstradas pelo número significativo de inscritos que desistiram do curso, semao menos realizar o primeiro acesso no ambiente virtual de aprendizagem (Rodrigues et al., 2018; Santos \& Oliveira Neto, 2009). Além disso, a evasão foi relativamente alta e pode ter sido ocasionada por não motivação, falta de integração social e interesses pessoais, além dos mesmos fatores que levaram à não participação (Reser \& Silva, 2018; Rodrigues et al., 2018).

Gonçalves (2021) assinala que a videoaula de curta duração gravada tem alto nível de eficácia no aprendizado em decorrência da flexibilidade e do engajamento. Os resultados da pesquisa de satisfação demonstraram que o formato de videoaulas foi aprovado pela maioria dos participantes. Em ambientes de EaD on-line, surgem formas específicas de comunicação, de ação e interação entre os sujeitos que podem influenciar diretamente os processos de aprendizagem. O tutor pode ter um papel decisivo nesse processo (Castro \& Mattei, 2008); entretanto, o curso não proporcionou interação entre tutor e alunos

Em 2006, a IAEA publicou o primeiro Nuclear Medicine Resources Manual, que traz orientação clara sobre necessidades de recursos humanos e treinamento para médicos, tecnólogos, cientistas radiofarmacêuticos, físicos e enfermeiros especializados na prática de MN (International Atomic Energy Agency [IAEA], 2006). Todavia, no Brasil, estudos sugerem que profissionais de enfermagem raramente são incluídos em programas de treinamento relacionados à proteção radiológica (Melo et al., 2015).

Em 2020, com a publicação do segundo NuclearMedicine Resources Manual, a IAEA destaca que muitos países têm dificuldade em compreender a importância da formação de tecnólogos que atuam em $\mathrm{MN}$ e, com isso, o desenvolvimento profissional deste grupo tem atrasado em relação aos outros. Como resultado, existem muitos tecnólogos trabalhando em medicina nuclear que tiveram pouco ou nenhum treinamento formal em $\mathrm{MN}$ e, a partir dessa realidade, a IAEA desenvolveu um programa de treinamento a distância. Tal situação foi corroborada pelos resultados dessa ação de extensão, já que as inscrições do curso foram interrompidas 15 dias antes do prazo devido à grande demanda de interessados no curso. 


\section{Conclusão}

O curso, promovido pela ação de extensão, proporcionou conhecimento sobre física aplicada e segurança em $\mathrm{MN}$, além de normas e gerenciamento em serviços de medicina nuclear à equipe multidisciplinar desses serviços. Dessa maneira, este estudo evidenciou a extensão como estratégia para capacitar os IOEs, garantindo a capacitação anual prevista em legislação brasileira, mesmo durante a pandemia da COVID-19.

Cabe destacar que o curso atingiu o seu objetivo, pois a maioria dos concluintes do curso afirmou descobrir novos métodos para melhorar determinadas atitudes e que mudará suas práticas, porquanto o curso ensinou diversos métodos de proteção radiológica a que eles passaram a dar importância.

A ação de extensão promoveu a interação entre discentes da educação superior, da pós-graduação, docentes e comunidade externa em nível nacional e internacional. Dessa maneira, ocorre a consolidação da cultura extensionista na educação superior e o despertar da pós-graduação para a necessidade de uma integração com a sociedade por meio da extensão. Entretanto, ações mais inclusivas ainda são neces sárias, principalmente quanto ao uso do AVA e participação ativa dos inscritos. Vislumbra-se, para ações futuras, além da ampliação do tempo do curso, a inclusão de um módulo específico para nivelamento no uso do AVA e promoção de encontros síncronos para suporte aos cursistas, pois acredita-se que, dessa forma, se minimizam as possibilidades de evasão. Assim, ratificam-se as ações de extensão como importantes mobilizadores de competências que transpassam os limites físicos das instituições de ensino.

\section{Agradecimentos}

Ao IFSC - Campus Florianópolis, pelo apoio por meio do EDITAL No 18/2019/PROEX/PROPPI/Câmpus Florianópolis.

\section{Con tribuição de cada autor}

Todos os autores participaram da redação do artigo e de todas as etapas descritas na metodologia.

\section{Referências}

Camozzato, T. S. C., Alves, A. S., Coutinho, A. G. F., Oliveira, C. V. de, Silva, C. da, Vargas, F. C. de, ... \& Prandi, T. M. D. (2019). Medicina Nuclear. In P. F. Dorow \& C. Medeiros (Orgs.). Proteção Radiológicano Diagnóstico e Terapia. (pp. 85109). Florianópolis: Publicações do IFSC. Recuperado de https://www.ifsc.edu.br/documents/30701/ 523474/PROTE\%C3\%87\%C3\%830+RADIOLO\%20GICA+ebook+final.pdf/10be750c-0d7c-484f-8baf-c33053f203cd

Cardenuto, R. M., Trilha, C. C. da S., Pacheco, F., Oliveira, M. M. de, \& Maciel, W. A. (2017). As atividades de extensão como compromisso social: Um estudo de caso no Instituto Federal de Santa Satarina (IFSC) e na Fundação Universidade do Estado de Santa Catarina (UDESC). Revista Gestão Universitária na América Latina-GUAL, 10(4), 134-156.

Castro, R. I. V. G. de, \& Mattei, G. (2008). Tutoria em EaD on-line: Aspectos da comunicação que favorecem a inte ração socioafetiva em comunidades de aprendizagem. Revista Brasileira de Aprendizagem Aberta e a Distância (RBAAD) 7, a208.

Comissão Nacional de Energia Nuclear - CNEN. (2013). Norma CNEN NN 3.05 - Requisitos de Segurança e proteção radiológica para serviço de medicina nuclear. Brasília: Ministério da Ciência, Tecnologia, Inovações e Comunicações. Recuperadode http://appasp.cnen.gov.br/seguranca/normas/pdf/Nrm305.pdf 
Gonçalves, R. (2021). EAD na UFRJ: eficácia, qualidade e satisfação. Avaliação de dois cursos de graduação no Instituto de Economia. Universidade Federal do Rio de Janeiro - UFRJ, Instituto de Economia, IE-UFRJ Discussion paper.

Gonçalves, TD 011 . Recuperado de https://www.ie.ufri.br/images/IE/TDS/2021/ TD IE 0112021 GONCALVES cAnexo.pdf

Granjeiro, E. M., Musse, J. O., Peixoto, T. M., Nunes, I. V., Soares, I. M. S. C., Silva, I. C. O., Carvalho, T. B., \& Dias, Y. O. (2020). Estratégias de ensino à distância para a educação interprofissional em saúde frente à pandemia COVID-19. Revisa, 9(Esp.1), 591-602.

International Atomic Energy Agency(IAEA). (2006). Nuclear Medicine Resources Manual. Vienna: IAEA.

International Atomic Energy Agency(IAEA). (2020). NuclearMedicine Resources Manual. 37. ed. Vienna: IAEA.

Instituto Federal de Educação, Ciência e Tecnologia de Santa Catarina (IFSC). (2014). PPGPR: Programa de PósGraduação em Proteção Radiológica. Florianópolis: IFSC. Recuperado de http://cs.ifsc.edu.br/portal/files/ FPOLIS MESTRADO PROTE\%C3\%87\%C3\%830 RADIOLOGICA PPC 997.pdf

Instituto Federal de Educação, Ciência e Tecnologia de Santa Catarina (IFSC). (2016). Resolução CONSUPno61, de 12 de dezembro de 2016. Regulamenta as Atividades de Extensão no IFSC. Boletim de ServiçolFSC, Florianópolis. Recuperado de http://cs.ifsc.edu.br/portal/files/consup resolucao61 2016 extensao.pdf

Instituto Federal de Educação, Ciência e Tecnologia de Santa Catarina (IFSC). (2018). PPC N03: Projeto Pedagógico do Curso superior em tecnologia em radiologia. Florianópolis: IFSC. Recuperado de http://www.florianopolis.ifsc.edu.br/ index.php?option=com content\&view=article\&id=979\&ltemid=188

Lisboa, R. C. S. N., \& Rendeiro, M. M. P. (2019). A aplicabilidade do desenho universal: Ressignificando o ambiente virtual de aprendizagem para a inclusão digital. In G. de C. Daltro, M. L. R. Santos, A. O. A., M. D. de Sousa, M. F. D. Passos, A. F. Lemos, E. N. Nascimento, \& A. C. P. Fraga (Orgs.) Práticas inovadoras da rede UNA-SUS: Experiências e desafios para a educação permanente dos trabalhadores do SUS. (pp. 137-152). Salvador: EDUFBA. Recuperado de https://repositorio.ufba.br/ri/bitstream/ri/30890/3/Praticas\%20inovadoras\%20da\%20rede\%20UNA-SUS-miolo-RI-.pdf

Melo, J. A. C. de, Gelbcke, F. L., Huhn, A., \& Vargas, M. A. de O. (2015). The work process in radiological nursing: Invisibility ofionizing radiation. Texto \& Contexto - Enfermagem, 24(3), 801-808.

Melo, J. A. C. de (2018). Organização do trabalho e desgaste dos trabalhadores de enfermagem em serviços de mediana nuclear. (Tese de Doutorado). Universidade Federal de Santa Catarina, Centro de Ciências da Saúde, Programa de PósGraduação em Enfermagem, Florianópolis.

Melo, J. A. C. de, Silva, C. da, Alves, M. L. de S., Machado, I. de S. M., Laurindo, M., \& Fin, A. P. C. (2020). Experiências docentes e discentes: extensão universitária na pandemia de COVID-19: projeto radiologia na comunidade, o uso da rede social e ambiente virtual de aprendizagem. Saberes Plurais: Educaçãona Saúde, 4(2), 49-60.

Musse, J. de O., Granjeiro, E. M., Peixoto, T. M., Silva, D. C. e, Almeida, T. R. O., Carvalho, T. B. de, Soares, I. M. S. C., \& Silva, I. C. O. da (2021). Extensão universitária e formação em saúde: Experiências de um grupo tutorial do PET-Saúde Interprofissionalidade. Revista Brasileira de Extensão Universitária, 12(1), 103-112.

Nascimento, A. V., Dias, A. de J. R., Leite, E. R., \& Vicente, R. (2018). Tipologia, causas e consequências de acidentes radiológicos na radiologia médica. [S. I.]: Instituto de Pesquisas Energéticas e Nucleares - IPEN-CNEN/SP (GRR-REL03/19).

Portela, T., Camozzato, T. S. C., Flôr, R. de C., Ribeiro, G., \& Melo, J. A. C. de. (2021). Occupational exposure in the working process of radiological nursing in nuclear me dicine. Journal of Radiology Nursing, 49(3), $246-253$.

Reser, M. R., \& Silva, C. H. (2018). Ambiente virtual de aprendizagem sobre adesão à farmacoterapia pelos usuários dos serviços de saúde do SUS para os profissionais de saúde. Saberes Plurais: Educaçãona Saúde, 2(1), 23-23.

Rodrigues, L. S., Gontijo, T. L., Cavalcante, R. B., Oliveira, P. P., \& Duarte, S. J. H. (2018). A evasão em um curso de especialização em Gestão em Saúde na modalidade a distância. Interface (Botucatu), 22(66), 889-901. 
Santos, E. M. dos, \& Oliveira Neto, J. D. de (2009). Evasão na educação a distância: Identificando causas e propondo estratégias de prevenção. Paideia - Revista Científica de Educação a Distância, 2(2).

Santos, G. M. T. dos, Reis, J. P. C. dos, Mérida, E. C., Rangel, E. L. F., \& Frich, A. A. (2020). Educação Superior: Reflexões a partir do advento da pandemia da COVID-19. Boletim de Conjuntura (BOCA), 4(10), 108-114.

Souza, E. P. de. (2020). Educação em tempos de pandemia: Desafios e possibilidades. Cadernos de Ciências Sociais Aplicadas, 17(30), 110-118.

Viana, E. (2018). Aplicabilidade do ${ }^{99 m}$ Tc-mibi no carcinoma dife renciado da tireoide com ${ }^{131}$ negativo. (Dissertação de Mestrado em Proteção Radiológica). Instituto Federal de Santa Catarina, Florianópolis. Recuperado de https://repositorio.ifsc.edu.br/bitstream/handle/123456789/312/Disserta\%C3\%A7\%C3\%A30\%20final.pdf?sequence= 1\&isAllowed=y

Como citar este artigo:

Camozzato, T. S. C., Grams, N., Soares, F. A. P., de Melo, J. A. C., Cardoso, I. A., \& da Silva, M. J. (2021). Educação continuada em serviços de medicina nuclear: Capacitação da equipe multidisciplinar em tempos de COVID-19. Revista Brasileira de Extensão Universitária, 12(3), 349-362. https://periodicos.uffs.edu.br/index.php/RBEU/article/view/ $\underline{12411 / \mathrm{pdf}}$ 